\section{LA COLABORACIÓN ENTRE LA AGENCIA ESPAÑOLA DE COOPERACIÓN INTERNACIONAL PARA EL DESARROLLO (AECID) Y EL MINISTERIO DE DEFENSA EN EL CAMPO DE LA AYUDA HUMANITARIA}

\author{
Gonzalo Vega Molina \\ Jefe de la Unidad de Prevención y Evaluación \\ Oficina de Acción Humanitaria. \\ AECID. MAEC.
}

THE COLLABORATION BETWEEN THE SPANISH AGENCY FOR INTERNATIONAL DEVELOPMENT COOPERATION AND THE MINISTRY OF DEFENCE IN THE FIELD OF HUMANITARIAN AID

\begin{abstract}
Humanitarian aid has been more significant in the last few years, Spain has become an important humanitarian donor. In this context, the AECID and the Ministry of Defence have developed a wide collaboration annually reflected in a Plan of Action. The range of activities includes the response to natural disasters, humanitarian demining and Afganisthan reconstruction.
\end{abstract}

KEY WORDS: Humanitarian help; humanitarian de-mining; military-civic co-operation; Agencia Española de Cooperación Internacional para el Desarrollo (AECID) (Spanish Agency of International Co-operation for Development); United Nations; good humanitarian donor-ship; leading plan for spanish co-operation; humanitarian action strategy of spanish co-operation; Armed Forces.

INTRODUCCIÓN

Son muchos los ámbitos de colaboración entre el Ministerio de Asuntos Exteriores y de Cooperación (MAEC) y el Ministerio de Defensa cuando pensamos en actuaciones en el exterior. Probablemente, es en la acción humanitaria donde esta colaboración es menos conocida aunque empieza a tener mayor visibilidad en los últimos tiempos. La Agencia Española de Cooperación Internacional para el Desarrollo es una agencia estatal vinculada al MAEC encargada de la gestión de la política española de cooperación para el desarrollo. La ayuda humanitaria forma parte de la Ayuda Oficial al desa-
RESUMEN: La ayuda humanitaria ha tomado gran relevancia en los últimos años y España se ha convertido en un importante donante humanitario. En este contexto, la AECID y el Ministerio de Defensa han desarrollado una amplia colaboración que se refleja anualmente con la firma de un Plan Operativo y cuyas actividades van desde la respuesta a los desastres naturales a la reconstrucción de Afganistán pasando por el desminado humanitario.

PALABRAS CLAVE: Ayuda humanitaria; desminado humanitario; cooperación cívico-militar; Agencia Española de Cooperación Internacional para el Desarrollo (AECID); Naciones Unidas; Good Humanitarian Donorship; Plan Director de la Cooperación Española; Estrategia de Acción Humanitaria de la Cooperación Española; Fuerzas Armadas.

rrollo (AOD) pero es diferente en sus objetivos y principios de los proyectos de desarrollo. Su principal objetivo es salvar vidas. A lo largo de estas páginas, no se pretende hablar en profundidad de las operaciones de mantenimiento de paz, muy relacionadas con el tema objeto del estudio pero fuera de las competencias de la AECID. En este artículo se definirá brevemente el concepto de acción humanitaria para luego describir varios ámbitos concretos de relación como son la ayuda humanitaria en Afganistán, las operaciones de respuesta a emergencias, o el desminado humanitario, entre otros. Este análisis permitirá conocer algunos de los futuros retos de la ayuda humanitaria española. 


\section{LA AYUDA HUMANITARIA: DEFINICIÓN Y NOTAS LEGALES}

La ayuda humanitaria puede definirse como el apoyo que los países se prestan en caso de catástrofe natural, aislada o más o menos permanente (como las sequias) y aquella que se da en el contexto de los conflictos armados. Su objetivo principal es salvar y proteger vidas humanas y restablecer sus condiciones de vida. La AECID dispone en 2008 de más de casi 120 millones de euros para acciones de ayuda humanitaria, tras incrementos sostenidos a lo largo de años anteriores. Además, España se ha incorporado a las más importantes iniciativas internacionales en la materia tanto de las Naciones Unidas (OCHA) como de la comunidad de donantes (Directrices de Oslo e Iniciativa del Good Humanitarian Donorship). España es además unos de los principales contribuyentes del Fondo Central de Respuesta a Emergencias de las Naciones Unidas (CERF en sus siglas en inglés), en el año 2008 con 30 millones de euros. La reforma de la $\mathrm{AECl}$ con el Decreto 1403/2007, de 26 de octubre, tiene como uno de sus pilares la creación de la Oficina de Acción Humanitaria, una oficina con más medios, presupuesto y clara separación de las tareas. La nueva Oficina de Acción Humanitaria diferenciará la prevención, la respuesta a emergencias y la evaluación.

Dicho esto, se debe señalar que la alta disponibilidad de las FFAA, sus capacidades y medios y, a fin de cuentas, el hecho de constituir un elemento fundamental del Estado hace que puedan ser muy útiles en la facilitación de la ayuda humanitaria y en la creación de espacios de seguridad, en un momento en que las organizaciones humanitarias sufren el acoso y la violencia de las partes en un conflicto armado o del terrorismo internacional.

Antes de entrar en cuestiones más concretas, resulta interesante hacer una breve referencia al marco normativo internacional y nacional.

En 2003 se publicaron los 23 principios de la Iniciativa de "Buena Donación Humanitaria" (Good Humanitarian Donorship -GHD-, en su traducción al inglés), una guia de buenos usos humanitarios a la que España se adhirió en el año 2004. Se trata de una iniciativa internacional que agrupa a los principales donantes humanitarios. En la actualidad, se encuentra en proceso de elaboración el Plan de acción español del GHD. Tres de estos principios tienen relación directa o indirecta con el papel de las FFAA en las operaciones de ayuda humanitaria:

-El principio 17: estar preparados para ofrecer apoyo al desempeño de acciones humanitarios, incluida la facilitación del acceso humanitario seguro.

- El principio 19: afirmar el papel primordial de organizaciones civiles en el desempeño de la $\mathrm{AH}$, particularmente en zonas afectadas por conflictos armados. En situaciones en que se utilizan la capacidad y los recursos militares para apoyar la $\mathrm{AH}$, garantizar que este uso está en conformidad con las leyes humanitarias internacionales y con los principios humanitarios y que reconoce la función de liderazgo de las organizaciones humanitarias.

-El principio 20: apoyar la aplicación de las Directrices sobre el Uso de Recursos de Defensa Militar y Civil para el alivio de Desastres de 1994 y las Directrices sobre el Uso de Recursos de Defensa Militar y Civil para Apoyar Actividades Humanitarias de Naciones Unidas en emergencias Complejas de 2003 (las conocidas como "Directrices de Oslo").

La Ley española de cooperación internacional para el desarrollo de 1998 señala que La cooperación española promoverá el respeto al derecho humanitario y asimismo apoyará en este ámbito medidas para la prevención y resolución de conflictos, incluyendo las operaciones de mantenimiento y consolidación de la paz, instrumentadas por medio de acuerdos bilaterales o multilaterales.

Según el Plan Director de la Cooperación Española 20052008, la acción humanitaria española se basará en el respeto profundo de los principios y valores humanitarios acordados internacionalmente y de acuerdo al Derecho Internacional. En este sentido, la dirección de toda acción exterior en la que se emplean tanto medios civiles como militares para la ayuda humanitaria corresponde al Gobierno, con especial referencia a la Secretaría de Estado de Cooperación Internacional y a su principal organismo operativo y de gestión, la AECID. El nuevo PD 2009-2012 contiene un capitulo específico y refuerza estas ideas.

El artículo 15.2 de la LO 5/2005, de Defensa Nacional, dice que las FFAA contribuyen militarmente a la seguridad y la defensa de España y de sus aliados, en el marco de las organizaciones internacionales de las que forma parte, así como el mantenimiento de la paz, la estabilidad y la ayuda humanitaria. 
La Estrategia de Acción Humanitaria de la Cooperación Española para el Desarrollo, publicada en 2008, menciona la especial capacidad del Ministerio de Defensa para movilizar recursos, sus características inherentes y su alta disponibilidad lo que le confieren un papel singular, cuando se requiere un despliegue inmediato de la ayuda humanitaria, principalmente en zonas donde la seguridad no está garantizada.

La Estrategia señala igualmente que en línea con las Directrices de Oslo y las recomendaciones del Comité de Ayuda al Desarrollo de la OCDE (CAD) la tendencia debiera ser que la participación de efectivos militares se circunscriba a las funciones de mantenimiento de la paz, creación de condiciones de seguridad y apoyo indirecto a la prestación de ayuda por parte de organizaciones imparciales y neutrales. Por lo que se refiere a la ayuda humanitaria prestada por parte del Estado, las FFAA constituyen un instrumento más del Estado que puede aportar un valor añadido mediante el uso de sus medios y capacidades. Se dará prioridad a los medios civiles excepto cuando por razones de oportunidad o eficacia se considere conveniente su empleo y su acción se valore como adecuada.

La Estrategia también afirma que se impulsará el establecimiento de una posición clara en materia de actuación militar, justificada por motivos humanitarios, que evite la confusión. Debería apoyarse más intensamente la implantación real en la ONU del principio de "responsabilidad de proteger".

En un esfuerzo por concretar estas cuestiones, el Consejo de Cooperación, órgano consultivo del gobierno en materia de cooperación al desarrollo que reagrupa a actores públicos y privados, ha puesto en marcha un grupo de coherencia de políticas encargado de estudiar, entre otras cuestiones, el papel de las fuerzas Armadas en las operaciones de ayuda humanitaria. En este grupo han participado representantes del MAEC y del Ministerio de Defensa.

\section{El Convenio Marco de Colaboración: EL DESMINADO HUMANITARIO}

Las relaciones entre la AECID y el Ministerio de Defensa se fundamentan en el Convenio Marco de colaboración firmado entre ambas partes en el año 1996. En su cláusula primera queda establecido que esta colaboración tiene lugar entre la Agencia y la Dirección General de Política de Defensa (DIGENPOL) para la ejecución y financiación de programas de cooperación internacional de interés mutuo y con carácter preferente a los dirigidos a aquellos países a los que prioritariamente convenga ayudar debido a razones de proximidad física, histórica o cultural. El Convenio Marco prevé además la creación de una Comisión de Coordinación y seguimiento que se reúna al menos una vez al año y la firma de Planes Operativos Anuales (POAs) en los que se determinen las acciones que han de realizarse $y$, en su caso, los medios personales o materiales necesarios.

Desde el año 1996, año de la firma del Convenio Marco, se han firmado seis POAs en los años 2002, 2004, 2005 2006, 2007 y 2008. El Plan Operativo para el año 2009, firmado el pasado 7 de enero, contempla la realización de un curso de operaciones de paz para los países de la zona iberoamericana y cuatro proyectos de formación en el área de desminado para países beneficiarios de la Cooperación Española como son Bosnia-Herzegovina, Líbano, Senegal, Jordania. Todos son países que todavía padecen las terribles consecuencias para sus poblaciones de la utilización de las minas antipersona.

En 1997 se firmó la Convención de Ottawa sobre la prohibición del empleo, almacenamiento, producción y transferencia de minas antipersonal y sobre su destrucción o Convención sobre la prohibición de minas antipersonal. La Convención señala diferentes obligaciones para los Estados firmantes como por ejemplo la destrucción progresiva de los arsenales existentes o la prohibición de seguir fabricándolas. Pero la Convención también incluye la obligación de que los Estados colaboren entre sí para lograr cumplir los objetivos marcados.

El desminado humanitario, componente esencial de las actividades relativas a las minas, abarca diversas actividades para la remoción de minas sin estallar. Éstas incluyen estudios técnicos, levantamientos cartográficos, remoción de minas y municiones sin estallar, señalización, documentación posterior al desminado y traspaso de las tierras desminadas (Normas internacionales para las actividades relativas a las Minas, IMAS). Se distingue del desminado militar pues su objeto es la posterior utilización del terreno desminado por parte de la población civil. 
Los cuatro cursos que la AECID y el Ministerio de Defensa organizarán en 2009 son cursos de instructores de desminado humanitario. Los cursos tienen lugar en el Centro Internacional de Desminado situado en la localidad madrileña de Hoyo de Manzanares. Los cursos están dirigidos a un máximo de 25 asistentes, con una duración máxima de cuarenta días. El objetivo es colaborar con dichos países en la formación teórico-práctica de sus técnicos para que puedan desarrollar con seguridad las tareas de desminado en su territorio. El Ministerio de Defensa facilita los medios e instalaciones para desarrollar el curso (materiales, instalaciones de enseñanza, instructores y profesorado así como el alojamiento para los alumnos entre otros). La AECID sufraga los gastos de pasaje aéreo, el per diem de los alumnos y su seguro sanitario. La AECID cuenta con la Fundación Internacional de Políticas Públicas y para Iberoamérica (FIIAPP) para la gestión de estos cursos.

Los cursos han tenido muy buen resultado y se incrementa la demanda año tras año.

El curso de Operaciones de Paz para Iberoamérica se enmarca en la prioridad dada por el Plan Director de la Cooperación Española 2005-2008 a la prevención de conflictos. El curso será impartido por expertos españoles a favor de 18 oficiales superiores de las Fuerzas Armadas de distintos países iberoamericanos. El curso permitirá la difusión de conocimientos sobre operaciones de mantenimiento de la paz, el intercambio de experiencias y el aprendizaje de procedimientos y documentos básicos de este tipo de operaciones.

España también financia el Fondo para actividades de desminado de las Naciones Unidas y diversos proyectos con ONGs.

\section{LA RESPUESTA EN SITUACIONES DE CONFLICTO ARMADO: El PRT en Afganistán}

La OTAN, bajo mandato de Naciones Unidas, tiene el mando de la fuerza multinacional de estabilización y ayuda a Afganistán (ISAF). España ha asumido el firme compromiso de contribuir a la reconstrucción y estabilización política de Afganistán mediante el apoyo a la ISAF. Además de sus funciones en el mantenimiento de la seguridad y estabili- dad, el mandato de ISAF incluye el desarrollo de programas de reconstrucción y la implantación de las instituciones del Estado en territorio afgano. Esto se está llevando a cabo a través de los Equipos de Reconstrucción Provincial (PRT en sus siglas en inglés) que contempla la incorporación de civiles para el cumplimiento de esta parte de su mandato.

España aporta unos 650 efectivos y lidera un PRT en la provincia de Badghis concretamente en la localidad de Qala i Nao, situada a $150 \mathrm{~km}$ por carretera de la ciudad de Herat. La provincia se sitúa al oeste del país y hace frontera con Turkmenistán. Se trata de una zona remota, aislada, pobre y atrasada, situada en un terreno montañoso y con unas condiciones climatológicas duras en invierno. La AECID aporta al componente civil un equipo de cooperación con carácter permanente y un equipo de ingenieros de la empresa pública TRAGSA. Está desplegado desde septiembre de 2005 para la puesta en marcha del programa de reconstrucción identificado previamente.

La cooperación española tiene como objetivo estratégico global en Afganistán la disminución de la pobreza, apoyando la reconstrucción, la mejora de las comunicaciones, mejorando el sistema sanitario, el desarrollo económico y social, fomentando la plena integración de los colectivos vulnerables en especial el de las mujeres e incidiendo en el desarrollo rural.

El Programa de Reconstrucción se basa en dos ideas principales: la provincia no presenta signos visibles de destrucción por la guerra pero sí una situación generalizada de pobreza y, además, no dispone de las mínimas infraestructuras básicas ni de materiales y maquinaria para llevarlo a cabo. Por todo ello, las áreas de actuación identificadas han sido las siguientes: agua potable y saneamiento, salud, comunicaciones por carretera, educación, regadíos, ayuda alimentaria y género. Se suma a esta lista el fortalecimiento institucional como línea horizontal de actuación.

Los signos más visibles de este ambicioso y completo programa de reconstrucción son el abastecimiento total de la población en agua potable, la rehabilitación y puesta a punto del hospital provincial de Qala i Nao, la rehabilitación de carreteras, la construcción de una pista de aterrizaje, la construcción de escuelas y la financiación al Programa Mundial de Alimentos. 
El Ministerio de Defensa proporciona la seguridad necesaria para los trabajos ejecutados por la AECID.

El último gran logro que cabe señalar se refleja en la publicación de un estudio sanitario de la Universidad Johns Hopkins de Baltimore que reconoce los avances de la provincia de Badghis en la detección y tratamiento de la tuberculosis. Según este estudio, Badghis sería la primera provincia de Afganistán en sistemas de detección y tratamiento de la tuberculosis. Se trata de un importante avance ya que la tuberculosis afecta a las capas de la población más vulnerables como las mujeres y los niños. Una de las razones del éxito, atribuible en gran parte a la cooperación española, ha sido seguir una línea de trabajo bajo los criterios sanitarios afganos.

\section{La cooperación cívico-militar}

La AECID también ha mantenido reuniones con el Ministerio de Defensa en relación con las actividades de cooperación cívico-militar (CIMIC). En este tipo de actividades los militares (formando unidades denominadas "CIMIC") parte de una operación de mantenimiento de la paz entran en contacto con la población de la zona en la que se desarrolla su operación para buscar soluciones a algunos problemas de la localidad como puede ser el mantenimiento o reparación de alguna infraestructura o la distribución de bienes. Se busca así crear lazos fuertes con la sociedad del lugar en el que trabajan. Con este tipo de actuación, el ejército busca mejorar su imagen de cara a la población lo que redunda en una mejor percepción de su misión y un incremento en el nivel de su seguridad. Estas actividades no pueden calificarse como de humanitarias pues no se rigen por sus principios (neutralidad, imparcialidad, independencia y humanidad) pero son útiles para el propósito que persiguen. En este punto hay que insistir en la idea de que se trata de actividades propias de los militares, diferentes de las que realiza la AECID y legítimas en sus objetivos e intereses.

El Ministerio de Defensa desarrolla este tipo de actividades en Afganistán y en Líbano. Según los datos que tiene la AECID, cuentan con un presupuesto de 60.000 euros al mes, lo cual no es una cantidad nada desdeñable. Son proyectos de corta duración, en torno a unos dos meses.

La AECID comprende las motivaciones que impulsan el desarrollo de este tipo de operaciones CIMIC pero es im- portante recordar que según las normas internacionales debe reafirmarse la necesaria separación en las funciones de cada institución.. En todo caso, la AECID está satisfecha del permanente diálogo entablado con el Ministerio de Defensa en estos asuntos y su gran disponibilidad. Este intercambio de información es un claro ejemplo de que la coordinación con el Ministerio de Defensa también funciona a la perfección en un asunto en el que los pareceres pueden ser distintos.

\section{El espacio humanitario}

Uno de los terrenos en el que la participación de las FFAA puede resultar indispensable es a la hora de asegurar el llamado "espacio humanitario". Hoy en día, el trabajo de las organizaciones humanitarias (Naciones Unidas, Comité Internacional de la Cruz Roja, ONGs...) está siendo cada vez más obstaculizado (asesinatos, secuestros, obstaculización del trabajo...). La violencia de un conflicto armado se dirige no sólo contra las partes enfrentadas sino también contra los actores que son independientes por definición y que trabajan en beneficio de la población más necesitada. La garantía de este trabajo depende en algunas situaciones de la protección que den los ejércitos de los países. En el caso español, las tropas que trabajan en países donde pervive un conflicto permiten con su presencia la continuación del trabajo de numerosas organizaciones

\section{LA RESPUESTA A DESASTRES NATURALES: LAS OPERACIONES DE ENVÍO DE AYUDA HUMANITARIA}

En caso de catástrofe natural de cierta entidad, la AECID pone siempre en marcha su protocolo de actuación en emergencias. Éste consiste básicamente en la toma de contacto y coordinación con los diferentes actores humanitarios, Ministerios, Comunidades Autónomas y ONGs, para poner en su conocimiento los datos de que se disponga. Esta toma de contacto se hace a través de correo electrónico o de mensaje en el teléfono móvil en el caso de producirse fuera del horario laboral. La información que la AECID tiene proviene de nuestras Embajadas y Oficinas Técnicas de Cooperación y de organismos internacionales como 0CHA (la oficina de Coordinación para los Asuntos Humanitarios de las Naciones Unidas), ECHO (la Oficina Humanitaria de la 
Comisión Europea) o de la Federación Internacional de la Cruz Roja.

En el caso de ser necesario, la AECID convoca a todos estos actores a una reunión de coordinación en la que, por un lado, se informa de la situación y, por el otro, se comparten la disponibilidad y capacidades de cada uno. La AECID también es responsable de diseñar y dirigir la respuesta que el Estado español pueda ofrecer. Siempre se tiene al corriente a la Embajada en España del país afectado y es habitual la participación del embajador/a al inicio de ésta. En estas reuniones, siempre se invita al Ministerio de Defensa.

Las relaciones entre la AECID y el Ministerio de Defensa en materia de respuesta a emergencias gira en torno al hangar que la AECID tiene en la base aérea de Torrejón de Ardoz para el envío de ayuda humanitaria por la vía aérea, la habitual. La nave cedida por el Ministerio de Defensa cuenta con un área de 800 metros cuadrados para el almacenamiento de material humanitario como mantas, tarpaulines (lonas de plástico de cobijo, tiendas de campaña, kits higiénicos, kits de cocina...). La gran ventaja es su ubicación junto a la pista, lo que brinda un acceso directo a la zona de carga de los aviones.

Existe un procedimiento a seguir para la utilización de la base por parte de la AECID que diferencia según se soliciten medios aéreos militares o simplemente el uso de la pista para un vuelo civil contratado. El intercambio de faxes entre ambos Ministerios incluye información acerca de las personas encargadas del operativo, la entrada de personal o la necesidad de contar con medios militares para el desplazamiento de la carga desde el almacén a la pista y posterior carga del avión. El Ministerio de Defensa ofrece numerosas facilidades como la entrega de permisos permanentes de entrada a la base para el personal de la AECID. También ha facilitado siempre la entrada de los medios de comunicación para que asistan a la carga del avión, puedan tomar imágenes del hangar y del material e incluso se celebren ruedas de prensa con la autoridades presentes.

En septiembre de 2007, tras el paso del huracán "Felix" por Nicaragua, el gobierno nicaragüense solicitó a España la financiación de medios aéreos muy necesitados para el transporte de la ayuda humanitaria entre la capital, Managua, y la zona damnificada, Puerto Cabezas. Tras las conversaciones mantenidas entre la AECID y el Ministerio de Defensa, se cedieron aviones Hércules pertenecientes al Ejército del Aire cuya utilización durante una semana permitió el establecimiento de este puente aéreo.

Otros ejemplos recientes de colaboración, en los que la ayuda humanitaria ha sido transportada en aviones del Ejército del Aire ha tenido lugar con motivo de las inundaciones de Tabasco en noviembre de 2007 o la ola de frío en Tayikistán en marzo de 2008 y terremoto Sichmán (mayo 2008).

En enero de 2008, la AECID ha abierto el Centro logístico Humanitario de la Cooperación Española en América Latina en Panamá para la atención de las crisis en ese continente, principalmente en América Central. La apertura de este Centro ha permitido ya dar respuesta a varias emergencias de manera más rápida, eficaz y menos costosa (inundaciones en la región del Beni en Bolivia, 2008), huracanes en el Caribe de septiembre 2008.

Es importante señalar que le uso de medios aéreos del ejército no sólo se ha producido en el marco de operativos de respuesta a catástrofes naturales, aunque es lo más habitual. También ha tomado protagonismo en algunos contextos de enfrentamiento armado como ocurrió en Líbano en el verano de 2006.

Cabe recordar, por último, que durante los dias 17 y 18 de noviembre de 2005 se celebró en la sede de la AECID el Seminario "La Colaboración entre las Organizaciones Humanitarias y las Fuerzas Armadas en la respuesta a los Desastres", organizado por el IECAH (Instituto de Estudios sobre Conflictos y Acción Humanitaria).

\section{ConcLusión}

Como se ha podido comprobar a la luz de los ejemplos mencionados, existe una buena relación, directa y de confianza, entre la AECID y el Ministerio de Defensa. Son muchas las áreas de trabajo en las que se puede obrar conjuntamente, sobre todo en aras de una buena gestión y resultado de las mismas.

El principio de unidad de acción en el exterior exige que la dirección de una acción de ayuda humanitaria que com- 
bine elementos civiles y militares corresponda siempre al Ministerio de Asuntos Exteriores y de Cooperación y, en concreto, a la Secretaría de Estado de Cooperación Internacional a través de la AECID.

En el futuro, el trabajo realizado en desminado humanitario y respuesta a diversos tipos de crisis deberá de perfeccionarse. La reforma de la $\mathrm{AECl}$ convertida desde enero de 2008 en AECID encuentra en la nueva Oficina de Acción Humanitaria uno de sus ejes fundamentales y una buena oportunidad para ello. La apuesta firme y decidida del gobierno por una ayuda humanitaria más eficaz, cuantiosa y de calidad debe ir acompañada por un fortalecimiento de la capacidad de la AECID para coordinar y liderar toda actuación española en este campo. España, una vez que se ha adherido a los principios del GHD y forma parte de las principales iniciativas internacionales en la materia, debe de impulsar su plena incorporación en los métodos de trabajo y en las relaciones entre el MAEC y el Ministerio de Defensa.

\section{BIBLIOGRAFÍA}

Plan Director de la Cooperación española 2005-2008, 2009-2012.

Estrategia de Acción Humanitaria de la Cooperación Española 2008.
Acuerdo marco entre el Ministerio de Defensa y la AECID 1996.

Plan Operativo AECID-Ministerio de Defensa 2009.
Recibido: 11 de enero de 2008 Aceptado: 17 de marzo de 2008 